Alex Bruce

Animal Law in Australia: An Integrated Approach

LexisNexis Butterworths (Chastwood, Australia 2018), 324 p.

ISBN 978-0-40934-380-9

\title{
Melanie Montenegro-Pérez
}

Bióloga especializada en Conservación de la Biodiversidad

Máster en Derecho Animal y Sociedad

Universidad Autónoma de Barcelona

Recepción: Junio 2018

Aceptación: Junio 2018

\section{Resumen}

El autor ha sabido plasmar de forma indiscutible las dificultades existentes en cuanto a la protección de los animales derivadas de la inconsistencia legislativa entre los diferentes Estados y Territorios que componen Australia. Esta situación se traduce en un sistema débil a la hora de garantizar el buen estado de bienestar de los animales.

Palabras clave: Australia, Alex Bruce, Bienestar Animal, Derecho Animal, Derecho Animal en Australia.

\section{Abstract}

The author has managed to capture indisputably the existing difficulties regarding the protection of animals derived from the legislative inconsistency between the different States and Territories of Australia. This situation translates into a weak system when it comes to ensure the good welfare state of the animals.

Keywords: Australia, Alex Bruce, Animal Welfare, Animal Law, Animal Law in Australia. 
El libro de Alex Bruce “Animal Law in Australia: An Integrated Approach” constituye una obra accesible y fundamental para entender la complejidad de la protección de los animales en Australia. Y es que la obra manifiesta de forma clara la inconsistencia y heterogeneidad de la legislación en cuanto a la protección de los animales en dicho país. Ante tal escenario, la capacidad de síntesis, recopilación, evaluación y comparación por parte del autor de la legislación existente en y entre los diferentes Estados y Territorios que conforman Australia queda patente a lo largo de toda la obra, que no podría ser más completa en tan sólo 324 páginas. La lectura del libro que nos ocupa resulta sumamente enriquecedora puesto que el aprendizaje personal va más allá de la adquisición de los conocimientos explicados en los capítulos. $\mathrm{Y}$ es que al final de cada uno de ellos se proporcionan diferentes fuentes adicionales por si el lector o la lectora desean ampliar la información recibida. La obra ofrece una visión panorámica de las diferentes formas en que los humanos tratamos a los animales y cómo la legislación existente, mediante excepciones e incoherencias, no asegura un buen estado de bienestar de los mismos. Si bien de acuerdo con el título del libro la mayoría de información hace referencia a la situación que se da en Australia, son numerosos los aspectos que pueden ser extrapolados a otros países. Y es que la obra abarca un amplio espectro de acciones, usos y vínculos entre el ser humano y el resto de animales, que se dan a nivel mundial. A continuación, se explican las ideas principales de cada capítulo.

El capítulo uno "Animal Law in History" (Derecho Animal en la Historia) establece la base argumental del trato que se daba en el pasado a los animales y permite, por lo tanto, entender el trato actual. El "movimiento de liberación animal” no es algo reciente, dado que desde la antigüedad han existido filósofos que eran partidarios de ofrecer un trato no cruel a los animales. El pensamiento histórico en relación a los animales y al uso que de estos hace el ser humano es diferente según la cultura y el país en cuestión. Este capítulo es esencial dado que establece las interacciones entre humanos y animales en el pasado, que eran el reflejo de la consideración que se tenía de los segundos y permite entender el contexto pasado y actual del trato hacia los mismos.

El capítulo dos "Development of Contemporary Animal Law" (Desarrollo del Derecho Animal Contemporáneo) evidencia la subjetividad en cuanto al significado de la idea de "liberación animal”, dado que para algunos filósofos como Peter Singer significa la posibilidad de explotar a los animales sin causar sufrimiento innecesario, mientras que para otros como Tom Regan la "liberación animal” ha de ser absoluta eliminando, por lo tanto, todo tipo de explotación.

El capítulo tres “Australian Legal and Regulatory Framework” (Marco legal y regulatorio australiano) es particularmente importante por cuanto permite conocer los principales problemas en relación al régimen regulador sobre los animales y su bienestar, que son los siguientes: 1) la Constitución no confiere poder directo al Gobierno australiano para aprobar legislación con respecto a los animales sino que este solamente tiene competencia para proponer Model Codes of Practices (MCOPs), estándares, recomendaciones y otros, que no tienen valor legal y cuya implementación está en mano de cada Estado y Territorio; 2) Australia es un país que considera a los animales como propiedad y 3) si bien todos los Estados y los Territorios tienen leyes anti-crueldad hacia los animales, existen numerosas leyes, regulaciones y políticas que a menudo se superponen o presentan duplicidades en el contenido, por lo que el régimen regulador es inconsistente y confuso; 4) la "Australian Animal Welfare Strategy" (AAWS) desarrollada por el "Department of Agriculture, Fisheries and Foresty" (DAFF) reconoce a algunos animales como seres sintientes y manifiesta que todos/as los/las australianos/as tienen el deber de garantizar que se mantiene y protege el buen estado de los animales y que no se les causa sufrimiento innecesario. No obstante, la AAWS no tiene fuerza de ley y cada Estado y Territorio tiene potestad para decidir si incorpora en su legislación lo establecido en la AAWS; 5) el hecho de que los MCOPs y los estándares de bienestar animal sean creados por comités cuyos componentes son representantes de departamentos que no tienen el bienestar animal como objetivo principal, lleva a un conflicto de intereses que impide una mayor protección de los 
animales.

El capítulo cuatro “Animals As Assistants” (Animales como asistentes) deja entrever, una vez más, los problemas asociados a que no exista regulación a nivel estatal, en esta ocasión en cuanto a los animales de asistencia. Según la legislación, existen prohibiciones acerca de la presencia de perros en determinados espacios, como jardines, parques, transportes públicos, taxis, aviones, restaurantes, incluso en pisos, pero también existen excepciones dentro de las cuales entran los animales de asistencia. En relación a los mismos, un ejemplo de la inconsistencia legislativa entre Estados y Territorios radica en las diferentes definiciones de "animal de asistencia” y "discapacidad", hecho que tiene como consecuencia la protección incompleta e insuficiente de las personas que dependen de animales de asistencia frente a actos de discriminación.

El capítulo cinco “Animals as Companions” (Animales como compañeros) muestra que Australia es uno de los países con la tasa más alta de animales de compañía (el $62 \%$ de los habitantes tiene animal de compañía) y que son numerosos los ataques de perros que suceden. En este sentido, cada Estado y Territorio tiene promulgadas leyes para regular los perros peligrosos o agresivos. No obstante, la aplicación real de estas leyes es llevada a cabo por los consejos locales, los cuales también han creado leyes que regulan a los perros en general y a los peligrosos en particular. A la existencia de diferentes niveles legislativos se le suma de nuevo la inconsistencia, en este caso en cuanto a la definición de "perro peligroso" y "ataque grave”. Más aún, el procedimiento de declaración de un perro como "peligroso" difiere entre Estados y Territorios, ya que en algunos la persona responsable del animal sólo puede apelar una vez escrita la declaración y en otros ni siquiera se le proporciona tal opción. El capítulo cinco también pone de manifiesto lo que representa para los animales ser considerados como "propiedad”. Y es que en Australia, cuando un animal de compañía muere por negligencia la compensación recibida es, normalmente, el valor del animal en el mercado.

En el capítulo seis “Animals as More Than Companions” (Animales como más que compañeros) se explica que, si bien en Australia son pocos los casos sobre custodia de animales de compañía cuando una pareja se separa, en otros países como Estados Unidos la decisión es tomada teniendo en cuenta los intereses de los animales. Asimismo, el autor también comenta que los responsables de animales de compañía pueden determinar en sus testamentos el futuro de los mismos, bien sea dejarlos a cargo de una persona de confianza, de una protectora o eutanasiarlos. No obstante, en cuanto a esta última opción, podría considerarse que va en contra de la legislación anti-crueldad de los Estados y Territorios de Australia si se trata de animales sanos. El síndrome de Noe y la zoofilia también son temas tratados en este capítulo. El síndrome de Noe consiste en la tenencia obsesiva de animales de compañía por parte de una persona que no puede cuidarlos adecuadamente debido a la numerosidad de los mismos y que, por lo tanto, deriva en negligencias graves y deterioración notable tanto de los animales como de la persona que sufre el trastorno. En este sentido, la "Royal Society for the Prevention of Cruelty to Animals" (RSPCA) confisca a los animales cuando se detecta y comunica un caso de síndrome de Noe. En cuanto a la zoofilia, es decir, la práctica de actividad sexual llevada a cabo por los humanos con los animales, también es objeto de inconsistencia legislativa, concretamente en cuanto a la definición, ya que en algunos Estados o Territorios, la zoofilia requiere que exista penetración. Las principales razones de prohibición de tal actividad en Australia es proteger la dignidad humana y prevenir los actos de crueldad hacia las personas y hacia los animales que no consienten la relación. El autor expresa que el bienestar de los animales implicados en tales actos es prácticamente pasado por alto en los debates sobre criminalización de la zoofilia.

El capítulo siete "Animals as Entertainment” (Animales como entretenimiento) abarca el uso de animales para su exhibición en zoológicos y en circos. Bruce explica que las funciones de educación, investigación y conservación de las especies que se asocian a los zoológicos hacen que el bienestar de los animales pase a un segundo plano. En el caso de los circos, el único argumento detrás de la exhibición de animales se encuentra en el 
entretenimiento de los espectadores. En cuanto al bienestar de los animales en los zoológicos y circos en Australia, no existe un régimen regulador consistente o único y es que, una vez más, este radica en estándares que el Gobierno propone y que los Estados y Territorios deciden si incorporan a su legislación sobre la materia relacionada. De hecho, algunos Territorios carecen de regulación específica sobre zoológicos y circos. Otro uso de los animales como entretenimiento son las carreras de caballos, muy populares en Australia y que, sin embargo, cuenta con escasa regulación y la existente, además, se centra en los beneficios económicos en lugar de en el bienestar de los caballos. Tampoco hay regulación uniforme sobre la participación de animales en películas y en obras de teatro.

El capítulo ocho "Animals and Cruelty" (Animales y crueldad) podría considerarse uno de los más relevantes dado que su lectura permite obtener una idea de la heterogénea situación entre Estados y Territorios. La tendencia de la crueldad hacia los animales en Australia está en aumento, siendo los domésticos el principal objetivo de tales acciones. Cada Estado y Territorio dispone de su legislación en cuanto a la prevención de la crueldad hacia los animales, si bien el título de la misma difiere. No obstante, se dan disimilitudes más importantes entre las legislaciones sobre el bienestar animal, dado que las prohibiciones acerca de la crueldad hacia los animales varían. Más aún, algunas leyes imponen deberes positivos con respecto a los animales, mientras que otras simplemente prohíben los actos de crueldad. Sin embargo, el establecimiento de excepciones para determinados animales (producción, experimentación, etc.) es común en todas las legislaciones. En cuanto a la definición de “animal”, la inconsistencia se hace notar una vez más. Y es que en algunos estados los peces no quedan recogidos dentro de tal definición, mientras que los anfibios y los reptiles, por ejemplo, sí.

El capítulo nueve “Animals as Food” (Animales como comida) constituye asimismo uno de los primordiales teniendo en cuenta que el consumo de animales es un hecho mundial. La producción de animales para el consumo humano en Australia muestra una tendencia de crecimiento durante los últimos años, siendo la carne de cerdo, de pollo y de vaca las más consumidas. La protección de los animales y de su bienestar en el sector alimentario es una ardua tarea dado que, como indica el autor, existe una falta de conexión por parte de los consumidores entre el producto final que se vende y su origen, es decir, un animal sintiente y los numerosos procesos a los que ha estado sometido, incluida la fase de sacrificio. Asimismo, la regulación de tal ámbito viene dada por el "Primary Industries Ministerial Council” (PIMC), cuyo objetivo es la creación de industrias de productos animales que sean rentables y explotables. Teniendo en cuenta que el PIMC forma parte del “Agriculture Minister's Forum” (AGMIN), la autoridad responsable de la creación de estándares de bienestar animal en Australia, el conflicto entre los intereses de los animales y el de los que elaboran las leyes es patente.

El capítulo diez “Animals as Pests” (Animales como plagas) ejemplifica de forma indiscutible cómo la inconsistencia legislativa vulnera la protección eficiente de los animales. $\mathrm{Y}$ es que en el caso de esta categorización de los animales tampoco existen disposiciones legislativas adecuadas. Esto, junto con la formulación de estándares y códigos de práctica no aplicables no hace sino permitir de forma indirecta el sufrimiento de los seres considerados como "plaga". Y es que, al no existir consistencia en la regulación en cuanto a los procedimientos a seguir para controlar a estos animales, los métodos crueles están permitidos, dejando en un estado de indefensión a los animales categorizados bajo el nombre que los condena.

La legislación australiana sobre el uso de animales para experimentación y educación es el tema del capítulo once “Animals and Science” (Animales y ciencia). Millones de animales son utilizados en investigación cada año en Australia. En cuanto a la regulación de estas actividades, si bien la legislación sigue siendo inconsistente en determinados aspectos entre Estados y Territorios, cabe destacar que esta presenta un denominador común: el principio de las tres $\mathrm{R}$ (Reemplazo de los animales por otros métodos, Reducción del número de ejemplares y Refinamiento, es decir, que los efectos 
negativos en el bienestar de los animales sean los mínimos posibles). La R de "reemplazo" es la que recibe menos importancia por parte de los responsables de elaborar la legislación, lo que refleja el posicionamiento a favor de la continuidad de la investigación con animales. No obstante, las comunidades científicas han reconocido una cuarta R (Responsabilidad de las industrias respecto a sus consumidores y al bienestar de los animales). Dadas las atrocidades que se cometen puertas adentro de los laboratorios, favorecidas por la consideración de los animales como "propiedad" y que el potencial fin de la experimentación con animales se halla lejos (al menos, hoy en día), las inconsistencias legislativas entre Estados y Territorios, también presentes en este ámbito, se agravan.

En el capítulo doce "Animals and International Law” (Animales y Derecho Internacional) el autor explica la dificultad a la hora de proteger a los animales derivada de las actividades internacionales tales como la importación y la exportación de animales vivos y la caza de ballenas. En cuanto a lo primero, una de las principales dificultades radica en asegurar que el manejo de los ejemplares en los países de destino favorece un buen estado de bienestar. Esta falta de control sobre lo que sucede con los animales una vez descargados constituye una de las principales debilidades en cuando a la legislación internacional que, en el caso de Australia, es más grave ya que es uno de los mayores exportadores de animales vivos. En cuanto a la caza de ballenas, se explica cómo Japón exprime la excepción sobre la caza con fines científicos para cazar en un santuario de ballenas situado en Australia. Asimismo, se expresan los problemas internos y externos que aparecen a la hora de aplicar lo establecido por la Convención sobre el Comercio Internacional de Especies Amenazadas de Fauna y Flora Silvestres (CITES) cuyas disposiciones fueron incorporadas en la legislación australiana. Por otro lado, resulta interesante la propuesta de Declaración Universal sobre Bienestar Animal que actualmente se encuentra en estado latente y que, si bien tendría carácter recomendatorio y no obligatorio, su aceptación supondría un paso importante de cara a reforzar el bienestar animal a nivel internacional.

El capítulo trece "Future Directions” (Direcciones futuras), como su título indica, expone los potenciales problemas futuros a los que la sociedad se enfrentará como consecuencia del aumento de la población, que llevará asociada la demanda de producción de carne y, por ende, un impacto en el bienestar de los animales destinados al consumo. Asimismo, la ciencia seguirá teniendo un papel fundamental a la hora de demostrar la capacidad de sentir de los animales y contribuir a la reforma de la legislación en pro de los mismos, asociada también a la evolución de la moral de la sociedad. El autor finaliza la obra manifestando su esperanza de haber aportado a las lectoras y a los lectores conocimientos necesarios para su trabajo e investigación personal en el campo del Derecho Animal.

De acuerdo con todo lo que precede, la conclusión que se extrae del libro es que, en Australia, el régimen regulador en relación a los animales es, como se ha podido percibir en la síntesis de los capítulos, inconsistente y heterogéneo. Esto es debido a los diferentes niveles legislativos y a la ausencia de una ley nacional sobre bienestar animal, lo que debilita la protección de los animales. Por lo tanto, es necesario un cambio del sistema legislativo en Australia, que haga más fuerte la protección de los animales mediante la harmonización de las leyes de bienestar animal y de los MCOPs entre Estados y Territorios. La creación de un departamento destinado específicamente al desarrollo de estándares adecuados de bienestar animal, así como a garantizar su aplicación, debe ser un objetivo de cara a los años venideros. “Animal Law in Australia: An Integrated Approach” debería considerarse una llamada urgente, un toque de atención al egocentrismo de los seres humanos. La lectura de esta obra es perentoria para entender que, a pesar de que los animales han formado, forman y formarán parte de la vida de los seres humanos y que aportan numerosos beneficios a los mismos, sus intereses siguen estando considerados por debajo de los de los humanos en todos los ámbitos. Por último, cabe recordar que, del largo camino que queda por recorrer dentro del Derecho Animal, eliminar la consideración de los animales como "cosas" o "propiedad" a nivel mundial debe ser una prioridad. 